$57^{\text {ème }}$ Congrès de la SFMBCB, 03003 (2011)

DOI:10.1051/sfmbcb/20115703003

(C) Owned by the authors, published by EDP Sciences, 2011

\title{
Carcinome épidermoïde sur syndrome LEOPARD : présentation d'un cas
}

\author{
Tabeti-Bentahar CF, Bouzouina F \\ Service de Pathologie et Chirurgie buccale, CHU, Oran, Algérie
}

Le syndrome LEOPARD (SL) est une maladie héréditaire rare dont la première description a été faite par Zeisler et Becker en 1936. C'est en 1969 que Gorlin et al. utilisent l'acronyme LEOPARD pour en désigner les différentes manifestations: L pour lentigines ou taches cutanées multiples, E pour anomalies de l'électrocardiogramme, O pour hypertélorisme oculaire, P pour sténose de l'artère pulmonaire; A pour anomalies de l'appareil génital, $\mathrm{R}$ pour retard de croissance et $\mathrm{D}$ pour surdité (deafness). A ce jour, environ 200 cas ont été répertoriés mais l'incidence réelle n'est pas établie (Sarkozy et al. 2008). Le SL est dû à une mutation du gène PTPN $11(80 \%$ des cas) ou du gène RAF 1. L'expression et la sévérité des symptômes sont variables mais sa pénétrance est importante.

Le cas rapporté est celui d'une fillette âgée de 13 ans, $4^{\text {ème }}$ enfant d'une fratrie de 6 enfants (4 garçons et 2 filles), née de parents non consanguins. La malade a été adressée en juin 2005 pour prise en charge odontostomatologique. Sur le plan général, elle présente un retard staturo-pondéral $(1,39 \mathrm{~m}$ pour $33 \mathrm{~kg})$ ainsi qu'une surdité. Aucune anomalie cardiaque n'est retrouvée. A l'examen somatique, des lentigines sont présentes sur l'ensemble du corps, associées à des taches café au lait, des nodules cutanés et une onychodystrophie. L'examen de la face révèle des lentigines multiples, un hypertélorisme, un strabisme convergent, une racine du nez large avec présence d'un naevus conjonctif sur l'aile narinaire droite. L'examen endobuccal retrouve un mauvais état buccodentaire et une langue dépapillée. Une radiographie panoramique et un bilan sanguin sont demandés avant la réalisation d'extractions multiples. La malade est perdue de vue et ne revient à la consultation qu'en novembre 2006. Elle présente alors une formation d'allure suspecte, en « chou-fleur », sur la lèvre inférieure. Il existe un retard de la maturité sexuelle. Le bilan sanguin objective une anémie (hémoglobine $6 \mathrm{~g} / \mathrm{dL}$ ) associée à une hypercalcémie $(112 \mathrm{mg} / \mathrm{L})$. La malade bénéficie d'un traitement chirurgical associé à une chimiothérapie et une radiothérapie. L'étude anatomopathologique de la pièce opératoire a confirmé le diagnostic de carcinome épidermoïde de la lèvre inférieure. La patiente est décédée en décembre 2007 des complications du carcinome alors que les malades atteints d'un SL décèdent le plus souvent de complications cardiovasculaires.

Le SL fait partie des syndromes «neuro-cardio-facio-cutanés ». C'est un syndrome rare, à transmission autosomale qui aurait une prédominance masculine (Jozwiak et al. 2009).

Voron et al. en 1976 ont proposé des critères diagnostiques du SL. En présence de lentigines, il faut l'association à 2 autres critères parmi les anomalies cutanées, cardiaques (structures ou électrocardiogramme), génito-urinaires, endocriniennes, neurologiques, squelettiques (dysmorphies cranio-faciales, petite taille). En l'absence de lentigines, 3 des critères sus-cités doivent être retrouvés. Le diagnostic différentiel se discute avec le syndrome de McCuneAlbright, le syndrome de Turner, le syndrome de Noonan, la neurofibromatose de type I, le syndrome de Moynahan et le syndrome de Costello. Les tumeurs malignes associées au SL décrites dans la littérature (Sarkozy et al. 2008) sont à type de mélanome malin, de myélodysplasie, de leucémie myéloïde et de neuroblastome ; la survenue d'un carcinome épidermoïde n’a jamais été rapportée.

Correspondance : tabeti_cherifa@yahoo.fr 